\title{
Adipose-derived lipocalin 14 alleviates hyperglycaemia by suppressing both adipocyte glycerol efflux and hepatic gluconeogenesis in mice
}

\author{
Jimmy Tsz Hang Lee ${ }^{1,2}$. Zhe Huang ${ }^{1,2} \cdot$ Kewu Pan ${ }^{1,2} \cdot$ Herbert Jialiang Zhang ${ }^{1,2}$. \\ Connie Waihong Woo ${ }^{1,3,4}$ Aimin $\mathrm{Xu}^{1,2,3,4} \cdot$ Chi-Ming Wong ${ }^{1,2,3,4}$
}

Received: 14 July 2015 / Accepted: 23 October 2015 / Published online: 23 November 2015

(C) Springer-Verlag Berlin Heidelberg 2015

\begin{abstract}
Aims/hypothesis Growing evidence supports that dysregulation of adipose tissue-derived factors contributes to the pathogenesis of diabetes and its complications. Since our global gene profiling analysis has identified lipocalin-14 (LCN14) - a secretory protein with lipid-binding properties - as a potential adipokine highly expressed in white adipose tissue (WAT), this study aims to explore the metabolic roles of LCN14 in obese mice, and to investigate the functional mechanisms involved. Methods Immunoassays and western blotting were performed to determine the circulating level and tissue distribution of LCN14, respectively. Recombinant adeno-associated virus (rAAV)-mediated gene delivery was used to overexpress
\end{abstract}

Jimmy Tsz Hang Lee and Zhe Huang contributed equally to this study.

Electronic supplementary material The online version of this article (doi:10.1007/s00125-015-3813-3) contains peer-reviewed but unedited supplementary material, which is available to authorised users.

Chi-Ming Wong

wispwong@hku.hk

1 State Key Laboratory of Pharmaceutical Biotechnology, Li Ka Shing Faculty of Medicine, The University of Hong Kong, Pokfulam, Hong Kong Special Administrative Region, People's Republic of China

2 Department of Medicine, Li Ka Shing Faculty of Medicine, The University of Hong Kong, 21 Sassoon Road, Pokfulam, Hong Kong Special Administrative Region, People's Republic of China

3 Department of Pharmacology and Pharmacy, Li Ka Shing Faculty of Medicine, The University of Hong Kong, Pokfulam, Hong Kong Special Administrative Region, People's Republic of China

4 Research Centre of Heart, Brain, Hormone and Healthy Aging, Li Ka Shing Faculty of Medicine, The University of Hong Kong, Pokfulam, Hong Kong Special Administrative Region, People's Republic of China
LCN14 in diet-induced obese (DIO) mice and the effects on glucose and lipid metabolism were examined.

Results LCN14 is expressed predominantly in WAT. Both circulating levels of LCN14 and its expression in adipose tissues are repressed in DIO and genetically inherited diabetic $(d b / d b)$ mice. Overexpression of LCN14 by rAAV-mediated gene delivery in DIO mice significantly increased insulin sensitivity in major metabolic tissues and ameliorated hyperglycaemia by inhibiting hepatic gluconeogenesis. The reduced hepatic glucose production is attributed to the suppressive effects of LCN14 on the expression of gluconeogenic genes and on glycerol efflux in adipocytes, possibly by reducing the expression of aquaporin-7.

Conclusions/interpretation Reduced LCN14 expression is involved in the pathogenesis of obesity-related metabolic dysregulation. LCN14 exerts its beneficial effects on glucose homeostasis and insulin sensitivity via its actions in both adipocytes and hepatocytes.

Keywords Adipokine - Aquaporin 7 - Glucose homeostasis · Glycerol efflux · Insulin resistance $\cdot$ Lipocalin $\cdot$ Lipocalin 14 . Obesity

$\begin{array}{ll}\text { Abbreviations } \\ \text { AFABP } & \text { Adipocyte fatty acid binding protein } \\ \text { AQP } & \text { Aquaporin } \\ \text { CLAMS } & \text { Comprehensive Lab Animal Monitoring System } \\ d b / d b & \text { Genetically inherited diabetic } \\ \text { DIO } & \text { Diet-induced obese } \\ \text { EWAT } & \text { Epididymal white adipose tissue } \\ \text { GAPDH } & \text { Glyceraldehyde-3-phosphate dehydrogenase } \\ \text { GFP } & \text { Green fluorescent protein } \\ \text { GTT } & \text { Glucose tolerance test } \\ \text { HFD } & \text { High-fat diet }\end{array}$

Abbreviations

AQP Aquaporin

CLAMS Comprehensive Lab Animal Monitoring System

$d b / d b$

DIO Diet-induced obese

EWAT Epididymal white adipose tissue

GAPDH Glyceraldehyde-3-phosphate dehydrogenase

GTT Glucose tolerance test

High-fat diet 


$\begin{array}{ll}\text { HFHC } & \text { High-fat high-cholesterol } \\ \text { ISO } & \text { Isoprenaline } \\ \text { ITT } & \text { Insulin tolerance test } \\ \text { LCN } & \text { Lipocalin } \\ \text { MUP1 } & \text { Major urinary protein-1 } \\ \text { OBP } & \text { Odorant-binding protein } \\ \text { PI3K } & \text { Phosphatidylinositol-3-kinase } \\ \text { PTT } & \text { Pyruvate tolerance test } \\ \text { qPCR } & \text { Quantitative real-time PCR } \\ \text { rAAV } & \text { Recombinant adeno-associated virus } \\ \text { STC } & \text { Standard chow diet } \\ \text { SVF } & \text { Stromal vascular fraction } \\ \text { SWAT } & \text { Subcutaneous white adipose tissue }\end{array}$

Introduction

Lipocalins are a large group of small secreted proteins that bind, transport and store small hydrophobic molecules such as lipids [1]. Thirty-seven lipocalins have been found in humans [2]. Despite low sequence homologies among lipocalins, their highly conserved tertiary structure provides a stable scaffolding to bind various small hydrophobic molecules [3]. Lipocalins exhibit diverse functions including involvement in the immune response and energy metabolism. For example, both adipocyte fatty acid binding protein (AFABP) and lipocalin-2 (LCN2) are predominantly produced in adipose tissues with levels increasing during the development of obesity. AFABP and LCN2 act as proinflammatory adipokines mediating toxic lipid-induced insulin resistance and vascular dysfunction [4-6]. Retinol binding protein 4 (RBP4) is also an adipokine associated with insulin resistance in obese participants by inducing hepatic gluconeogenesis and insulin resistance of muscle [7]. On the other hand, major urinary protein-1 (MUP1) and lipocalin-13 (LCN13) are beneficial lipocalins that are mainly produced in liver. MUP1 regulates glucose metabolism by inhibiting hepatic gluconeogenesis, and promoting energy expenditure in skeletal muscle $[8,9]$. LCN13 regulates glucose metabolism by suppressing hepatic gluconeogenesis and stimulating glucose uptake in adipocytes, and lipid metabolism by suppressing hepatic lipogenesis and increasing fatty acid beta-oxidation $[10,11]$.

Adipose tissue is the key organ to store excess energy in the form of triacylglycerols. The number and size of adipocytes can be increased substantially. The extent of fat storage can vary largely, from $5 \%$ to $\sim 60 \%$ of total body weight in humans [12]. During fasting, triacylglycerols are hydrolysed into glycerol and NEFA through lipolysis in adipose tissues and released as critical precursors for hepatic gluconeogenesis and beta-oxidation, respectively $[13,14]$. In addition to its function as a lipid reservoir, adipose tissue is also an endocrine organ [15]. Change in adiposity affects the expression profile of adipose genes including those coding for adipokines [16, 17]. For example, adiposity is negatively correlated with the production of adiponectin, a well-known adipokine with antiinflammatory and insulin-sensitising properties [18-20].

In this study, we characterised the function of a putative lipocalin gene named Lcn 14 (also known as Obp2b) in glucose and lipid metabolism. The recombinant adenoassociated virus (rAAV)-mediated gene delivery approach was used to overexpress LCN14 in diet-induced obese (DIO) mice and its effect on the overall metabolic fitness was examined. Our results indicate that LCN14 is a novel metabolic regulator and its deficiency contributes to obesityrelated glucose dysregulation.

\section{Methods}

Animal studies All animal experimental protocols were approved by Animal Ethics Committee. Male C57BL/6N (Charles River Laboratories, Wilmington, MA, USA), C57BLKS $/ \mathrm{J}-\mathrm{m}^{+} /$Lepr $^{\mathrm{db}}$ (lean) and C57BLKS/J-Lepr ${ }^{\mathrm{db}} /$ Lepr $^{\mathrm{db}}$ $(d b / d b)$ mice (The Jackson Laboratory, Sacramento, CA, USA) were housed in our laboratory animal unit under controlled temperature $\left(23 \pm 1^{\circ} \mathrm{C}\right)$ with free access to water and food. The 8-week-old male mice were divided into two groups and fed on either standard chow diet (STC) or high-fat diet (HFD; 45\% energy from fat, Research Diets, New Brunswick, NJ, USA). Each group was randomised into two subgroups and i.v. injected with rAAV encoding either green fluorescent protein (GFP) or LCN14. The high-fat high-cholesterol (HFHC) diet contained $41 \%$ energy from fat and $0.21 \%$ cholesterol (D12079B, Research Diets). Glucose, glycerol, insulin and pyruvate tolerance tests were conducted as previously described [21]. Mice were i.p. injected with isoprenaline and in vivo lipolysis was performed to estimate glycerol efflux [22].

rAAV-mediated gene delivery system for LCN14 expression To construct rAAV vectors for overexpression of LCN14 or GFP, cDNA encoding mouse LCN14 or GFP was inserted into rAAV2-LSP1 vector [23]. To package rAAV, HEK293 cells were co-transfected with p5E18-VD2/8 and pXX6. Viral particles were purified by polyethylene glycol/aqueous twophase partitioning method [24], and were quantified by quantitative real-time PCR (qPCR). The primer sequences for Lcn14 were 5'-GTACACACAGGCCATGGTGTC-3' (sense), and 5'-TGTACTTGTGAGGCTCGTCAG-3' (antisense).

Biochemical and immunological analysis Total RNA from tissues was extracted with Trizol reagent (Invitrogen, Carlsbad, CA, USA), and cDNA was synthesised using cDNA synthesis kit (Promega, Madison, WI, USA). The primer sequences for qPCR can be found in previous publications [25-27]. Total cellular proteins were separated by SDS-PAGE and probed 
with antibody against LCN14 $(0.2 \mu \mathrm{g} / \mathrm{ml}), \mathrm{LCN} 13$ $(0.2 \mu \mathrm{g} / \mathrm{ml})$, adiponectin $(0.2 \mu \mathrm{g} / \mathrm{ml})$, phosphorylated Akt (pAktSer473 [0.2 $\mu \mathrm{g} / \mathrm{ml}]$; Antibody and Immunoassay Services, The University of Hong Kong), Akt $(1 \mu \mathrm{g} / \mathrm{ml})$, phosphorylated S6K (pS6KThr398 [1 $\mu \mathrm{g} / \mathrm{ml}]$ ), S6K $(1 \mu \mathrm{g} / \mathrm{ml}), \beta$-actin $(1 \mu \mathrm{g} / \mathrm{ml}), \beta$-tubulin $(1 \mu \mathrm{g} / \mathrm{ml})$, FLAG (1 $\mu \mathrm{g} / \mathrm{ml})$, glyceraldehyde-3-phosphate dehydrogenase (GAPDH $[1 \mu \mathrm{g} / \mathrm{ml}]$ ) (Cell Signaling, Beverly, MA, USA), or aquaporin-7 (AQP7 [1 $\mu \mathrm{g} / \mathrm{ml}]$ Merck Millipore, Billerica, MA, USA).

Serum glycerol levels were determined using the Free Glycerol Reagent (F6428, Sigma-Aldrich, St Louis, MO, USA). Stromal vascular fraction (SVF) and adipocytes were isolated from epididymal fat pads of 10 -week-old male C57BL/6N mice as previously described [28]. Adipocyte differentiation was induced as previously described [29]. Epididymal white adipose tissue (EWAT) from HFD-fed male mice was isolated for the ex vivo glycerol efflux assay [22]. Glycerol kinase activity was determined by a radioisotopic assay for phosphorylation of $\left[{ }^{3} \mathrm{H}\right]$ glycerol $[30,31]$ and hepatic glycogen level was measured by an acid-hydrolysis method [32].

\section{Production of polyclonal antibody against mouse LCN14} in rabbits The anti-mouse LCN14 polyclonal antibody was generated by immunisation of New Zealand female rabbits using endotoxin-free recombinant LCN14 purified from E. coli as described [21]. Anti-mouse LCN14 IgG was purified from the serum of immunised rabbits with protein $\mathrm{G}$ beads followed by affinity chromatography with recombinant mouse LCN14 as the ligand.

\section{Measurement of serum LCN14 by in-house ELISA} Affinity-purified anti-LCN14 IgG was biotinylated with a sulpho-NHS-biotin kit (Pierce, Rockford, IL, USA), and the biotinylated $\operatorname{IgG}$ was used as the detection antibody. Unlabelled anti-LCN14 IgG was used as the capture antibody for coating a 96-well microtitre plate overnight at $4^{\circ} \mathrm{C}$ at a concentration of $1 \mu \mathrm{g} / \mathrm{ml}$. On the next day, the content was completely discarded. The plates were washed three times with $1 \times$ PBS containing $0.1 \%$ Tween-20 and blocked with $1 \%(1 \mathrm{~g} / 100 \mathrm{ml}) \mathrm{BSA}$ in PBS for at least $3 \mathrm{~h}$. Mouse serum samples were diluted in 1\% BSA in PBS, pH 7.4. Then $100 \mu \mathrm{l}$ of the diluted samples or recombinant LCN14 standards were applied to each well, and the mixture was incubated at room temperature for $2 \mathrm{~h}$. The plates were washed three times with $1 \times$ PBS containing $0.1 \%$ of Tween-20, $\mathrm{pH} 7.4$ and then incubated with $100 \mu \mathrm{l}$ of the detection antibody $(0.15 \mu \mathrm{g} / \mathrm{ml})$ for $1 \mathrm{~h}$ at room temperature. After washing three times, the wells were incubated with streptavidin-conjugated horseradish peroxidase for $20 \mathrm{~min}$ and subsequently reacted with tetramethylbenzidine reagent for $15 \mathrm{~min}$; then $50 \mu \mathrm{l}$ of $0.5 \mathrm{M}$
$\mathrm{H}_{2} \mathrm{SO}_{4}$ was added to each well to stop the reaction, and the absorbance was measured at $450 \mathrm{~nm}$.

Indirect calorimetry and body composition The Comprehensive Lab Animal Monitoring System (CLAMS; Oxymax, Columbus, OH, USA) was used to measure food and water intake, volume of oxygen consumption, carbon dioxide, energy expenditure and physical activity. Body composition (fat mass and lean mass) was measured using ${ }^{1} \mathrm{H}$ magnetic resonance spectroscopy (Bruker BioSpin, Billerica, MA, USA) as described previously [21].

Statistical analysis All analyses were performed with GraphPad Prism (version 6.0; GraphPad Software, La Jolla California USA, www.graphpad.com). The data were presented as means \pm SE of three independent experiments with triplicates. Statistical significance was determined by one-way ANOVA, ANCOVA or Student's $t$ test. In all statistical comparisons, a $p$ value $<0.05$ was used to indicate a significant difference.

\section{Results}

LCN14 is an adipokine Increased adiposity is associated with change of gene expression in adipose tissues and hence induces insulin resistance and metabolic dysregulation [16, 17]. Therefore, we compared the gene expression profile in subcutaneous white adipose tissue (SWAT) obtained from age-matched mice fed with STC and HFD for 20 weeks, and found that the transcript of a putative lipocalin gene, $L \mathrm{cn} 14$, was downregulated in DIO mice (Fig. 1a). On the other hand, consistent with a previous report [33] the expression of $F g f 21, A f a b p$ (also known as Fabp4) and Lcn 2 mRNA in adipose tissue was increased in HFD-fed mice (Fig. 1b, electronic supplementary material [ESM] Fig. 1a-c). To determine the tissue distribution, qPCR analysis with cDNA from various tissues was performed (Fig. 1c). Our data revealed that Lcn 14 mRNA was mainly detected in various WATs, including perirenal white adipose tissue (PWAT), EWAT and SWAT. The tissue distribution pattern of $L c n 14$ is similar to that of Afabp and Lcn2 (ESM Fig. 1d, e) but not to that of $L c n 13$ (also known as Obp2a) or Mup1 (ESM Fig. 1f,g).

To explore whether Lcn 14 mRNA is translated into protein, polyclonal LCN14 antibody was raised. The specificity of $\alpha$-LCN14 antibody was examined by western blotting (Fig. 1d, ESM Fig. 2a) with lysates from HEK293 cells transfected with carboxyl-terminally FLAG-tagged LCN14. The size of LCN14 is in agreement with the predicted molecular mass $(20 \mathrm{kDa})$. LCN13, which shares the highest degree of homology (53\% identity) with LCN14, was used to test the specificity. Anti-LCN14 antibody only detected LCN14 in the 

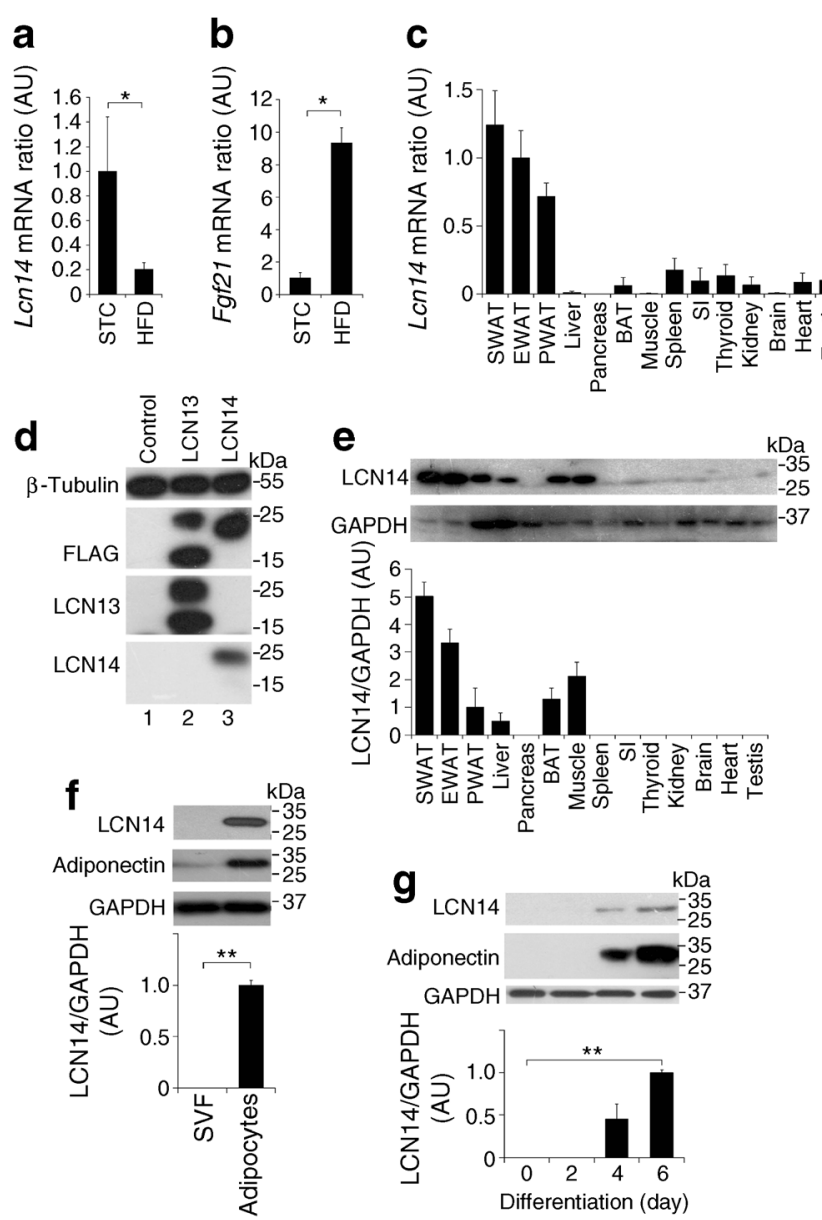

Fig. 1 LCN14 is mainly expressed in WAT. qPCR analysis was performed to measure the ratio of $L c n 14$ (a) and $F g f 21$ (b) mRNA expression in SWAT of 20-week-old STC or HFD-fed mice. The abundance of Lcn 14 mRNA in various tissues from 8-week-old STC-fed mice was measured by qPCR (c). Fold change was calculated relative to EWAT and was normalised against mRNA abundance of Gapdh. The rabbit anti-mouse LCN14 polyclonal antibody was generated to detect the presence of LCN14 (d). Immunoblot analysis of the abundance of LCN14 in various tissues of 8-week-old male mice normalised to GAPDH (e). LCN14 protein is expressed in adipocytes but not in SVF (f). Adipocytes and SVF were isolated from 10-week-old STC-fed mice. LCN14 protein was expressed in mature adipocytes from differentiated 3T3-L1 cells (g). Abundance of LCN14 was calculated relative to GAPDH adipocytes/differentiated 3T3-L1 cells. ${ }^{*} p<0.05,{ }^{* *} p<0.01$ for each group. $(n=5-8)$. AU, arbitrary units; PWAT, perirenal white adipose tissue; SI, small intestine

lysates of HEK293 cells overexpressing FLAG-LCN14, but did not react with FLAG-LCN13 (Fig. 1d, ESM Fig. 2a).

Tissue distribution of endogenous LCN14 in mice was then examined. Consistent with qPCR data (Fig. 1c), LCN14 was mainly expressed in WATs (Fig. 1e). LCN14 was predominantly found in mature adipocytes rather than SVF as demonstrated by cell fractionation (Fig. 1f) and differentiation of 3T3-L1 cells (Fig. 1g), with a pattern similar to that of the adipogenesis marker adiponectin [34]. LCN14 is predicted to be a secretory protein with amino acid residues 1-19 encoding a signal
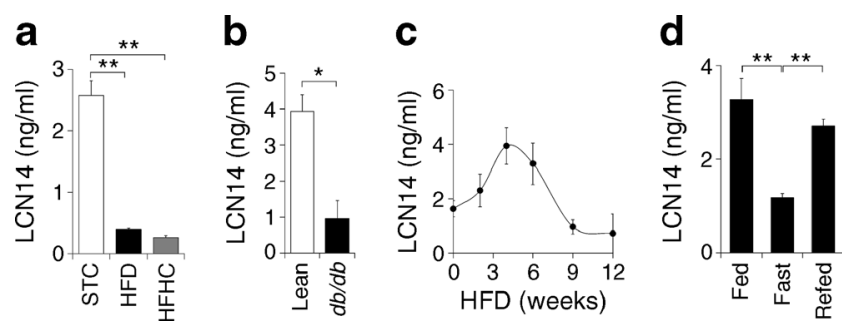

Fig. 2 Circulating LCN14 level is tightly regulated by nutrient status. LCN14 sandwich ELISA was used to quantify serum LCN14 levels in age-matched mice fed on STC, HFD or HFHC for 35 weeks (a) and lean and $d b / d b$ mice (b). Dynamic change of serum LCN14 levels in HFD-fed mice was monitored at different time points as indicated (c). Serum level of endogenous LCN14 in mice in fed state, after $14 \mathrm{~h}$ fasting and after refeeding for $6 \mathrm{~h}(\mathbf{d}) .{ }^{*} p<0.05,{ }^{*} p<0.01$. $(n=5-8)$

peptide and a trace amount of LCN14 was detected in tissues without Lcn14 mRNA expression (Fig. 1c, liver and muscle), suggesting that LCN14 may act as an adipokine to exert its metabolic action.

Circulating LCN14 is tightly regulated by nutrient availability LCN14 sandwich ELISA was developed (ESM Fig. 2b). The circulating LCN14 level in 39-week-old STC-fed male mice was $\sim 2.5 \mathrm{ng} / \mathrm{ml}$ (Fig. 2a). Consistent with the qPCR data (Fig. 1a), the circulating LCN14 level was downregulated by $\sim 4.5$-fold to below $0.5 \mathrm{ng} / \mathrm{ml}$ in mice fed on HFD or HFHC (Fig. 2a). The LCN14 level was also downregulated in genetically inherited diabetic $(d b / d b)$ mice (Fig. 2b). A time course study showed that the circulating LCN14 level was induced shortly after HFD treatment with the peak $(\sim 4 \mathrm{ng} / \mathrm{ml})$ at week 4 and then gradually decreased to a low level $(\sim 0.5 \mathrm{ng} / \mathrm{ml})$ after week 9 (Fig. 2c). Interestingly,

Fig. 3 HFD-fed rAAV-Lcn14 mice exhibit exacerbated hyperglycaemia and hepatic insulin resistance. Age-matched male mice were fed on either STC or HFD for 10 weeks followed by i.v. injection of rAAV encoding Lcn14 or Gfp. Fed and $14 \mathrm{~h}$ fasted blood glucose levels (a) were measured at the time points indicated. Fed (b) and $14 \mathrm{~h}$ fasted (c) serum insulin concentrations were measured by insulin sandwich ELISA. GTT (d, e) (D-glucose, $2 \mathrm{~g} / \mathrm{kg}$ body weight), ITT (f, g) (insulin, $0.4 \mathrm{IU} / \mathrm{kg}$ body weight) and PTT (h, i) (sodium pyruvate, $1 \mathrm{~g} / \mathrm{kg}$ body weight) were performed at weeks 6,7 and 8 after infection, respectively. The liver isolated from rAAV-Lcn14 mice and control mice after overnight fasting, followed by i.v. injection with insulin ( $4 \mathrm{IU}$ insulin/kg body weight) 10 weeks after infection, were subjected to western blotting for phosphorylated Akt (Ser473), total Akt and phospho-S6K (Thr389) (j). Relative amounts of p-Akt or p-S6K were determined by densitometry, normalised to total Akt and S6K, and are indicated at the bottom of the panels. The rAAV-Gfp group without insulin treatment was used as a control for comparison of fold differences. qPCR for mRNA levels of key gluconeogenic, lipogenic and beta-oxidation related genes in liver after $14 \mathrm{~h}$ fasting $(\mathbf{k})$. mRNA levels were normalised against abundance of $\beta$-actin and were calculated relative to GFP control. ${ }^{*} p<0.05,{ }^{* *} p<0.01$. $(n=6-9)$. White bar or dashed line with white squares, STC-fed rAAV-Gfp; dark grey bar or solid line with black squares, STC-fed rAAV-Lcn14; light grey bar or dashed line with white circles, HFD-fed rAAV-Gfp; black bar or solid line with black circles, HFD-fed rAAV-Lcn14 

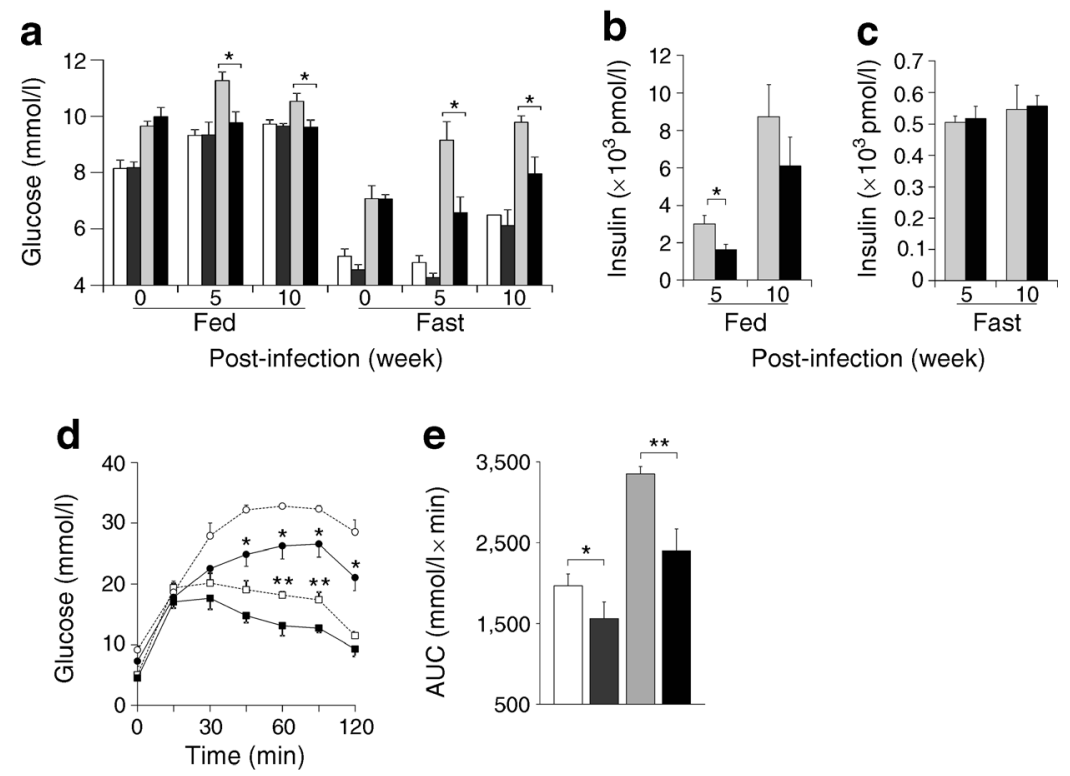

f

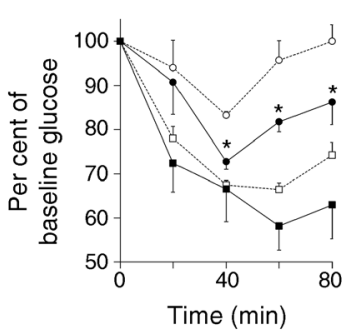

g
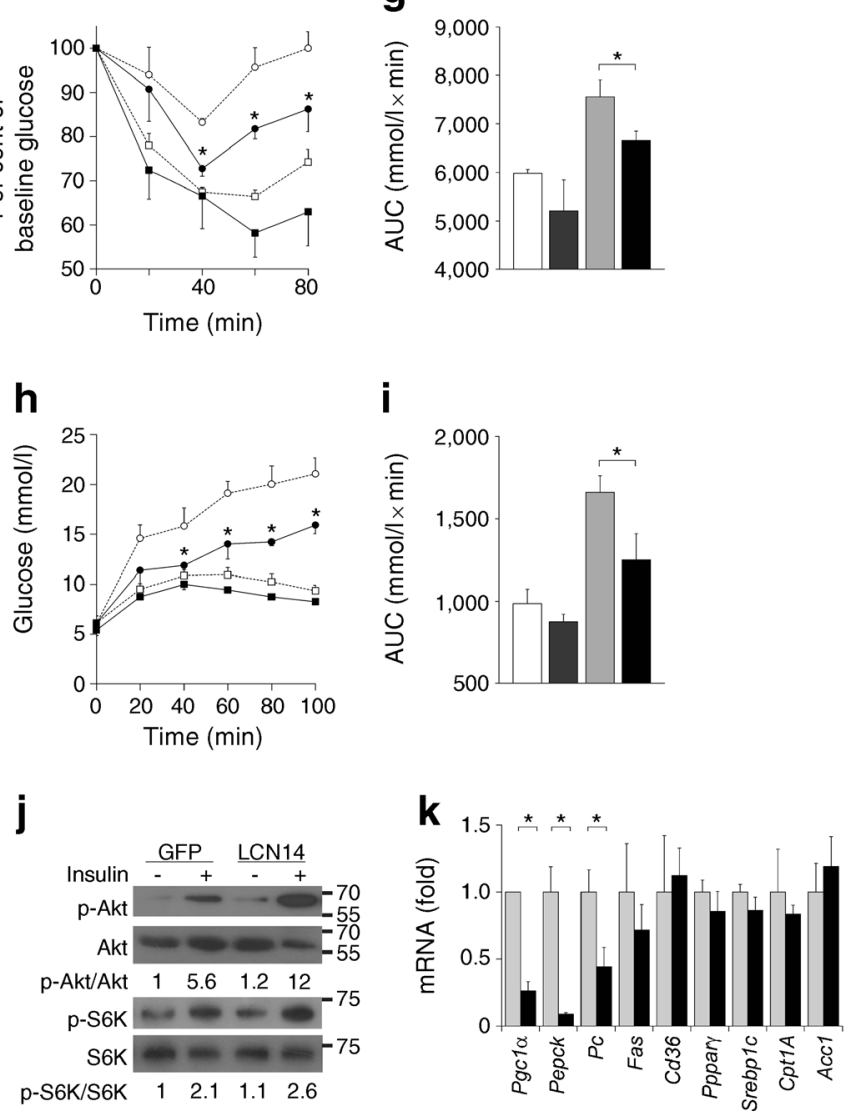

the circulating LCN14 level was altered in response to fed-fast cycle (Fig. 2d). Compared with feeding state, circulating LCN14 level was decreased by 2.5 -fold to $1 \mathrm{ng} / \mathrm{ml}$ after $14 \mathrm{~h}$ of fasting, and elevated to the normal level after $6 \mathrm{~h}$ of refeeding. Collectively, the circulating LCN14 level is tightly regulated by nutritional statuses.
Overexpression of LCN14 in DIO mice attenuated metabolic dysregulation caused by HFD Based on its expression pattern, we hypothesised that LCN14 deficiency may be involved in the pathogenesis of obesity. To evaluate the impacts of LCN14 on metabolism, LCN14 was overexpressed in DIO mice by rAAV-mediated gene expression system. FLAG-tagged LCN14 was expressed in liver and the 
circulating levels were analysed by western blotting (ESM Fig. 3a) and ELISA (ESM Fig. 3b). Overexpression of LCN14 did not affect caloric intake (ESM Fig. 3c), body weight gain (ESM Fig. 3d), body composition (ESM Fig. 3e,f) or energy expenditure (ESM Fig. 4a-c). However, respiratory exchange ratio (RER) in rAAV-Lcn14 mice was significantly higher than control in HFD-fed mice (ESM Fig. 4d,e).

Improved glucose homeostasis in rAAV-Lcn14 obese mice Overexpression of LCN14 did not alter the fed glucose level in STC-fed mice, but caused lower glucose level in HFD-fed mice under both fed and fasting conditions (Fig 3a). The serum insulin levels were reduced in HFD-fed rAAV-Lcn14 mice after feeding (Fig. 3b), but not in the fasting state (Fig. 3c). Improved glucose tolerance was observed in HFD-fed rAAV-Lcn 14 mice as revealed by glucose tolerance test (GTT) (Fig. 3d,e). The insulin tolerance test (ITT) showed that glucose levels in rAAV-Lcn 14 mice remained low at $40 \mathrm{~min}$ after injection of insulin as compared with the control (Fig. 3f, g). Furthermore, overexpression of LCN14 significantly decreased hepatic glucose production induced by sodium pyruvate in the pyruvate tolerance test (PTT) (Fig. 3h,i).

To determine whether overexpression of LCN14 enhances insulin signalling in liver of HFD-fed mice, fasted mice were euthanised after injection of PBS or insulin followed by examination of the activation of the Akt signalling pathway by measuring the phosphorylation levels of Akt at Ser473 and its downstream target S6K at Thr389. Insulin-stimulated Akt and S6K phosphorylation in liver samples from rAAV-Lcn 14 mice was more dramatically increased than the control (Fig. 3j), suggesting the insulin-sensitising effects were enhanced by LCN14 in liver.

The expression of key enzymes involved in hepatic gluconeogenesis, lipogenesis and beta-oxidation of rAAV-Lcn 14 mice was explored by qPCR (Fig. 3k). Overexpression of LCN14 could significantly inhibit hepatic gluconeogenesis by downregulating $\operatorname{Pgcl} \alpha$ (also known as Ppargcla), Pepck (also known as Pck1) and $P c$ (also known as $P r o c$ ), but did not affect the expression of lipogenic (Fas, Cd36, Ppary [also known as Pparg] and Srebp1c [also known as Srebfl]) and betaoxidation genes (Cptl $\alpha$ [also known as $\mathrm{Cpt1}$ ] and $\mathrm{Accl}$ [also known as Acaca]), suggesting that LCN14 lowered blood glucose level partially by suppressing hepatic gluconeogenesis.

\section{Suppression of circulating glycerol levels by LCN14 The} circulating lipid profile was measured. Surprisingly, although serum triacylglycerol (Fig. 4a) and NEFA (Fig. 4b) levels between the two groups were similar, the glycerol levels of rAAV-Lcn14 mice were significantly reduced by $\sim 12 \%$ and $\sim 17 \%$ under both fed and fasting conditions, respectively (Fig. 4c).
Adipose triacylglycerols are hydrolysed and released as glycerol and NEFA into the blood under fasting [35]. Unlike NEFA, due to low expression level of glycerol kinase in adipocytes, released glycerol cannot be effectively reutilised by adipose tissue; we therefore hypothesised that LCN14 might limit the supply of adipose glycerol for hepatic gluconeogenesis [36]. To test our hypothesis, mice were challenged with the nonselective $\beta$-adrenergic agonist isoprenaline (ISO) to stimulate adipose glycerol production through lipolysis [22]. Circulating glycerol level in rAAV-Lcn14 mice was increased by $58 \%$ at 16 min after injection, which was significantly lower than the respective level in the control (Fig. 4d,e). Consistently, the glucose level induced by isoprenaline in rAAV-Lcn14 mice was $\sim 30 \%$ lower than the control (Fig. 4f,g).

To confirm that the decrease in circulating glycerol level was not due to hepatic glycerol uptake or impaired hepatic glycerol kinase activity, exogenous glycerol was injected into the mice, and the circulating glycerol and glucose levels were measured [37]. The glycerol levels of both groups reached $\sim 100 \mu \mathrm{g} / \mathrm{ml}$ at $30 \mathrm{~min}$ and returned to basal levels at $180 \mathrm{~min}$ after injection (Fig. 4h). No significant difference in glucose level was seen between the two groups (Fig. 4i). In addition, treatment of primary hepatocytes with LCN14 did not affect their glycerol kinase activity (Fig. 4j). This finding further supports our hypothesis that rAAV-Lcn 14 mice can effectively utilise glycerol as a substrate in hepatic gluconeogenesis.

To further confirm the accumulation of glycerol in adipose tissues, weights of liver and adipose depots, as well as glycerol content in liver and EWAT were measured. Although no difference in their weights was observed (Fig. 5a,b), a lower amount of glycerol in liver and higher amount in EWAT were found in rAAV-Lcn14 mice vs the control (Fig. 5c). In addition, in agreement with previous studies [30,31], accumulation of glycerol in adipocytes treated with LCN14 was associated with the activation of adipose glycerol kinase activity (Fig. 5d). As increased intracellular glycerol content of adipose tissues would enhance adipose glycerol kinase enzymatic activity and accelerate the converting rate of intracellular glycerol to triacylglycerol in adipocytes, size of adipocytes and triacylglycerol levels from EWAT of the mice were measured. Consistent with our finding, the size of adipocytes (Fig. 5e) and triacylglycerol levels (Fig. 5f) of rAAV-Lcn14 mice were slightly higher than those of the control, although there was no statistical significance.

Decreased glycerol efflux from adipose tissues is due to improved insulin sensitivity and decreased expression of aquaporin-7 As previously reported, insulin is a key regulator in adipose glycerol efflux and hepatic gluconeogenesis during caloric deprivation [38]; the level of Akt phosphorylation in EWAT was thus examined by western blotting. LCN14 
Fig. 4 rAAV-mediated expression of LCN14 inhibits the glycerol efflux from adipose tissues in mice. Circulating lipid profile of the mice $(\mathbf{a}-\mathbf{c})$.

Triacyglycerol (a), NEFA (b) and glycerol (c) levels in rAAV-Lcn14 mice were measured under fed and 14-h fasting conditions at the indicated time points after injection. White bars, STC-fed AAV-Gfp; dark grey bars, STCfed AAV-Lcn 14; light grey bars, HFD-fed AAV-Gfp; black bars, HFD-fed AAV-Lcn 14. Change of plasma glycerol (d, e) and glucose levels (f, $\mathbf{g})$ after i.p. injection of isoprenaline $(1 \mathrm{mg} / \mathrm{kg}$ body weight). Glycerol clearance test (h, i) was performed by i.p. injection of exogenous glycerol ( $1.5 \mathrm{~g} / \mathrm{kg}$ body weight). The change of plasma glycerol (h) and glucose (i) levels was measured after injection. Primary hepatocytes of male mice treated with HFD for 10 weeks were isolated, followed by LCN14 (black bar) or PBS (white bar) treatment for $24 \mathrm{~h}$. Hepatic glycerol kinase activity was measured (j). ${ }^{*} p<0.05$. ( $\left.n=6-9\right)$. Light grey bars or dashed line with white circles, HFD-fed AAV-Gfp; black bars or solid line with black circles, HFD-fed AAVLcn 14. TG, triacyglycerol
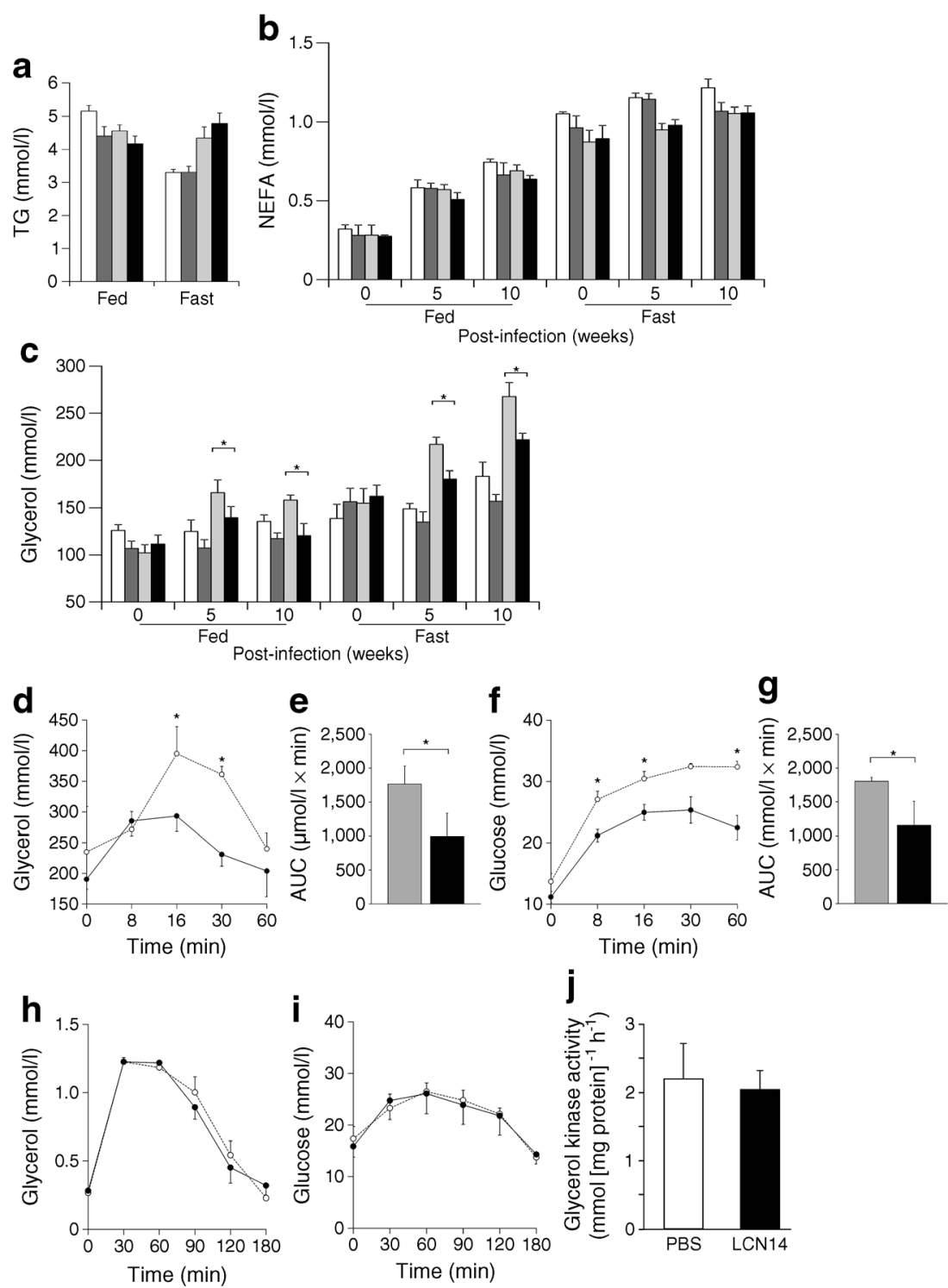

promoted the phosphorylation of Akt in EWAT (Fig. 5h) by insulin stimulation. Interestingly, the expression of aquaporin7 (AQP7), the key aquaglyceroporin that facilitates the secretion of glycerol in adipocytes [39], was repressed in EWAT of rAAV-Lcn 14 mice (Fig. 5h). This finding suggests that the alleviation of hyperglycaemia in rAAV-Lcn14 mice could also be attributed to the effect of LCN14 in lowering serum glycerol levels via inhibition of glycerol efflux from adipose tissues.

As the endogenous LCN14 level in EWAT of obese mice is low (Figs 1,2) and triacylglycerol content is high, an ex vivo lipolysis experiment with EWAT explants from obese mice was performed to explore whether LCN14 can directly inhibit glycerol release. Consistent with in vivo findings, LCN14 inhibited adipose glycerol efflux (Fig. 5i), but not NEFA release (Fig. 5j). Insulin was used as a positive control that lowers glycerol and NEFA release by inhibiting lipolysis [22]. As NEFA release was not affected by the treatment of LCN14 (Fig. 5j), we believed that the decrease of glycerol release was mainly due to the decrease in glycerol efflux instead of inhibition of lipolysis.

Previous studies have suggested that glycerol efflux of adipocytes is regulated by AQP7 via phosphatidylinositol-3kinase (PI3K) [40-42]. The PI3K inhibitor wortmannin was used to block the repression of adipose glycerol efflux by LCN14. Wortmannin increased both basal and isoprenalineinduced glycerol efflux [40-42]. Most importantly, wortmannin could alleviate the repression of isoprenalineinduced glycerol efflux by LCN14 (Fig. 5i). This suggests that LCN14 directly represses glycerol release from adipocytes probably via $\mathrm{AQP7}$ and the PI3K signalling pathway. 

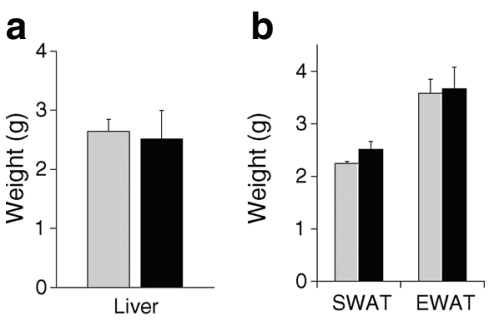

$\mathbf{e}$

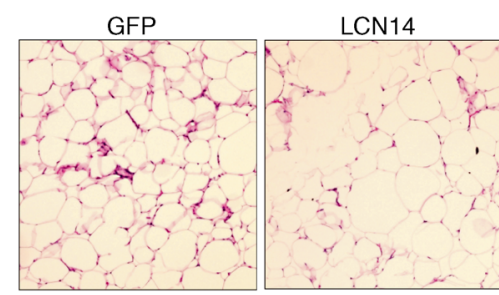

$$
1.8+0.5 \times 10^{3} \mu \mathrm{m}^{2}
$$

h
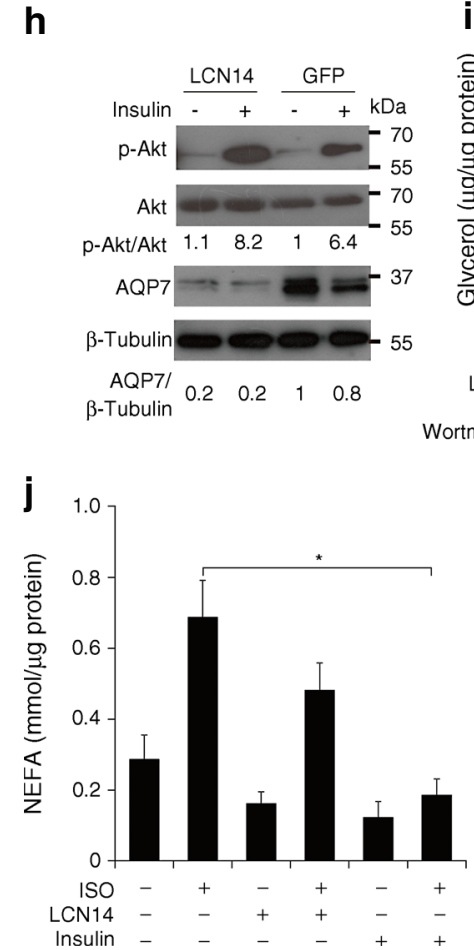

Fig. 5 LCN14 inhibits adipose glycerol efflux via PI3K. Weight of liver (a) and adipose tissue depots (b) and glycerol content in liver and EWAT (c) from rAAV-Gfp (light grey bars) and rAAV-Lcn14 mice fed with HFD (black bars) $(n=9)$. Fat explants from EWAT of male mice treated with HFD for 10 weeks were isolated, followed by LCN14 (black bars) or PBS (white bars) treatment for $24 \mathrm{~h}$. Adipose glycerol kinase activity was measured (d). Representative images of haematoxylin and eosin staining for EWAT (e) and triacyglycerol (TG) levels in EWAT (f) and liver (g) were measured. The indicated average size of the adipocytes was qualified by ImageJ software (http://imagej.nih.gov/ij/) as described previously [48]. Glycerol and TG levels were normalised to protein. rAAV-Lcn 14 mice and control were fasted overnight and i.v. injected with insulin (4 IU insulin $/ \mathrm{kg}$ body weight) in week 10 post-infection. Western blotting of EWAT of the mice was performed with phosphorylated Akt (Ser473), total
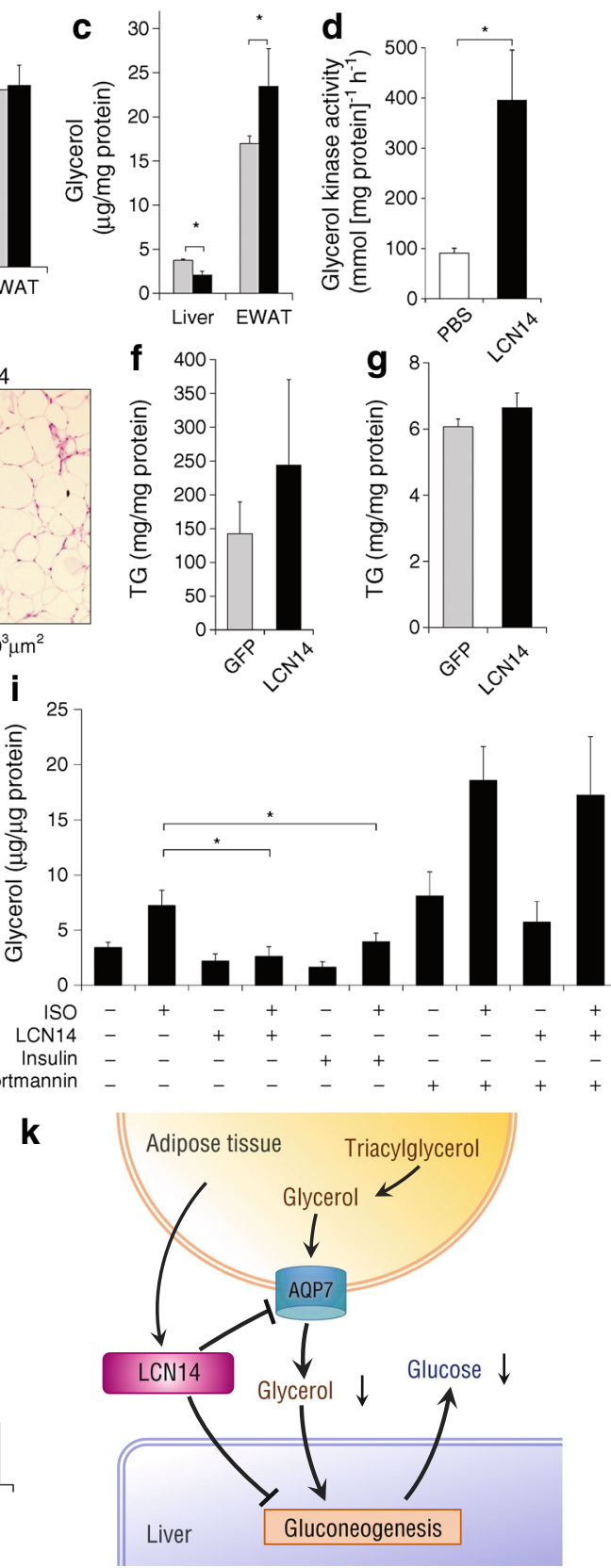

Akt and AQP7 antibodies (h). Relative amounts of p-Akt or AQP7 were determined by densitometry, normalised to total Akt and $\beta$-tubulin, and indicated below the blots. The rAAV-Gfp group without insulin treatment served as a reference. The fat explants from EWAT of male mice treated with HFD for 10 weeks were isolated, followed by LCN14, insulin or vehicle pre-treatment for $4 \mathrm{~h}$. Lipolysis was then induced by $1 \mu \mathrm{mol} / 1$ isoprenaline for $30 \mathrm{~min}$. Glycerol (i) and NEFA (j) concentrations in medium were measured and normalised to total protein content. Wortmannin $(10 \mu \mathrm{mol} / \mathrm{l})$ was used to treat the EWAT for $24 \mathrm{~h}$ before treatment with LCN14, insulin and isoprenaline as described in (i). ${ }^{*} p<0.05(n=6-9)$. A schematic representation of the proposed model describing the role of LCN14 in inhibiting hepatic gluconeogenesis via modulation of glycerol metabolism (k) 


\section{Discussion}

This study provides in vivo and in vitro evidence showing that the hypothetical lipocalin gene Lcn 14 encodes an adipokine with beneficial effects on metabolic dysfunction. LCN14 is predominantly expressed in mature adipocytes and its expression level is tightly regulated by metabolic states (Figs 1,2). Overexpression of LCN14 in DIO mice significantly alleviates diet-induced hyperglycaemia (Fig. 3) by lowering adipose glycerol efflux through downregulation of AQP7 (Fig. 5h), and improves insulin sensitivity of liver and adipose tissues (Figs 3j, 5h).

The beneficial effect of LCN14, however, cannot be solely explained by the limitation of glycerol efflux as deletion of Aqp7 did not improve insulin sensitivity of major metabolic tissues [43]. Previous studies demonstrated that HFD-fed Aqp 7 knockout mice developed hyperglycaemia and insulin resistance $[30,38,39]$. This may be due to the fact that accumulation of adipose glycerol in Aqp 7 knockout mice is much higher than the rAAV-Lcn14 mice [43, 44], and the beneficial effects of LCN14 in DIO mice are contributed by multiple mechanisms in different metabolic tissues including liver and adipose tissues (Figs 3, 5). It would be worthwhile to further explore how LCN14 regulates the expression of AQP7 in adipose tissues. The ligands and receptors for LCN14, as well as its intracellular signalling pathways also need to be explored in the future.

To sum up, we hypothesise that, under physiological conditions, LCN14 expression in WAT is high, which may inhibit adipose glycerol release during feeding. The expression of LCN14 was downregulated to promote the adipose glycerol release for hepatic gluconeogenesis during fasting. In obesity, the expression of LCN14 is repressed, AQP7 is overexpressed [45] and impaired insulin action in adipose tissues leads to increased lipolysis [46]. Consequently, the adipose glycerol efflux increases, more glycerol is available for hepatic gluconeogenesis and contributes to hyperglycaemia (Fig. 5k).

Previous studies demonstrated that its homologue LCN13 regulates glucose metabolism by enhancing insulin sensitivity in adipocytes and suppressing hepatic glucose production [10, 11]. Our study showed that LCN14 has similar functions in the attenuation of hyperglycaemia by increasing insulin sensitivities in liver (Fig. 3) and WAT (Fig. 5). However, in contrast with LCN13, LCN14 was not observed to improve hepatosteatosis (Fig. 5g), as LCN14 does not alter the expression of hepatic beta-oxidation and lipogenesis genes (Fig. 3k). In addition, LCN14 is mainly expressed in adipose tissues (Fig. 1c), whereas LCN13 is expressed in liver (ESM Fig. 1f) [11]. In this connection, LCN14 and LCN13 may alleviate hyperglycaemia by overlapping and distinct pathways. Furthermore, we found that rAAV-Lcn14 mice have increased locomotor activity by CLAMS (ESM Fig. 4f-h). Therefore, the central role of LCN14 remains to be determined.
LCN14 has two human orthologues, odorant-binding protein (OBP) $2 \mathrm{~A}$ and OBP2B [47], that share $62 \%$ and $63 \%$ similarity, respectively. With our in-house ELISA, both OBP2A and OBP2B can be detected in the serum of healthy non-obese middle-aged Asian individuals at concentrations of $46.0(32.3-65.5) \mathrm{pg} / \mathrm{ml}$ and $28.7(10.4-39.6) \mathrm{pg} / \mathrm{ml}$, respectively, suggesting that these orthologues of LCN14 are also present in the bloodstream of humans.

In conclusion, our study has identified LCN14 as a novel adipokine that can alleviate hyperglycaemia in DIO mice by improving the liver and adipose tissue insulin sensitivity, inhibiting hepatic gluconeogenesis and limiting glycerol release from WAT.

Acknowledgements The authors thank R. Hoo, K. Cheng, H. Hui and D. Ye (The University of Hong Kong, Hong Kong, China) for helpful discussion and critical reading of the manuscript; I. Alexander (The University of Sydney, NSW, Australia) for the AAV vectors and related plasmids; and P. Guo (University of Pittsburgh, Pittsburgh, PA, USA) for the protocol on purification of AAV.

Funding This work was supported by Collaborative Research Fund (C7055-14G) of Hong Kong Research Grant Council, internal research grant from the University of Hong Kong, and State Key Laboratory funding from The Innovation and Technology Commission

Duality of interest The authors declare that there is no duality of interest associated with this manuscript.

Contribution of authors All authors made significant contributions to the creation of this manuscript including conception and design, acquisition of data, analysis and interpretation of data, drafting and revising this article. AX and CMW developed the research question and study design. CMW is responsible for the integrity of the work as a whole. All authors have approved the final version for publication.

\section{References}

1. Flower DR (1996) The lipocalin protein family: structure and function. Biochem J 318(Pt 1):1-14

2. Du ZP, Wu BL, Wu X et al (2015) A systematic analysis of human lipocalin family and its expression in esophageal carcinoma. Sci Rep 5:12010

3. Akerstrom B, Flower DR, Salier JP (2000) Lipocalins: unity in diversity. Biochim Biophys Acta 1482:1-8

4. Kralisch S, Fasshauer M (2013) Adipocyte fatty acid binding protein: a novel adipokine involved in the pathogenesis of metabolic and vascular disease? Diabetologia 56:10-21

5. Jang Y, Lee JH, Wang Y, Sweeney G (2012) Emerging clinical and experimental evidence for the role of lipocalin-2 in metabolic syndrome. Clin Exp Pharmacol Physiol 39:194-199

6. Wu G, Li H, Zhou M et al (2014) Mechanism and clinical evidence of lipocalin-2 and adipocyte fatty acid-binding protein linking obesity and atherosclerosis. Diabetes Metab Res Rev 30:447-456

7. Kotnik P, Fischer-Posovszky P, Wabitsch M (2011) RBP4: a controversial adipokine. Eur J Endocrinol 165:703-711 
8. Hui X, Zhu W, Wang Y et al (2009) Major urinary protein-1 increases energy expenditure and improves glucose intolerance through enhancing mitochondrial function in skeletal muscle of diabetic mice. J Biol Chem 284:14050-14057

9. Zhou Y, Jiang L, Rui L (2009) Identification of MUP1 as a regulator for glucose and lipid metabolism in mice. J Biol Chem 284:1115211159

10. Sheng L, Cho KW, Zhou Y, Shen H, Rui L (2011) Lipocalin 13 protein protects against hepatic steatosis by both inhibiting lipogenesis and stimulating fatty acid beta-oxidation. J Biol Chem 286: 38128-38135

11. Cho KW, Zhou Y, Sheng L, Rui L (2011) Lipocalin-13 regulates glucose metabolism by both insulin-dependent and insulinindependent mechanisms. Mol Cell Biol 31:450-457

12. Kredel LI, Siegmund B (2014) Adipose-tissue and intestinal inflammation - visceral obesity and creeping fat. Front Immunol 5: 462

13. Fruhbeck G (2005) Obesity: aquaporin enters the picture. Nature 438:436-437

14. Perry RJ, Camporez JP, Kursawe R et al (2015) Hepatic acetyl COA links adipose tissue inflammation to hepatic insulin resistance and type 2 diabetes. Cell 160:745-758

15. Balistreri CR, Caruso C, Candore G (2010) The role of adipose tissue and adipokines in obesity-related inflammatory diseases. Mediat Inflamm 2010:19

16. Ronti T, Lupattelli G, Mannarino E (2006) The endocrine function of adipose tissue: an update. Clin Endocrinol 64:355-365

17. Rosen ED, Spiegelman BM (2006) Adipocytes as regulators of energy balance and glucose homeostasis. Nature 444:847-853

18. Wang Y, Zhou M, Lam KS, Xu A (2009) Protective roles of adiponectin in obesity-related fatty liver diseases: mechanisms and therapeutic implications. Arq Bras Endocrinol Metabol 53: 201-212

19. Cheng KK, Lam KS, Wang B, Xu A (2014) Signaling mechanisms underlying the insulin-sensitising effects of adiponectin. Best Pract Res Clin Endocrinol Metab 28:3-13

20. Hui X, Gu P, Zhang J et al (2015) Adiponectin enhances coldinduced browning of subcutaneous adipose tissue via promoting M2 macrophage proliferation. Cell Metab 22:279-290

21. Wong CM, Wang Y, Lee JT et al (2014) Adropin is a brain membrane-bound protein regulating physical activity via the NB3/Notch signaling pathway in mice. J Biol Chem 289:25976-25986

22. Choi SM, Tucker DF, Gross DN et al (2010) Insulin regulates adipocyte lipolysis via an Akt-independent signaling pathway. Mol Cell Biol 30:5009-5020

23. Cunningham SC, Dane AP, Spinoulas A, Logan GJ, Alexander IE (2008) Gene delivery to the juvenile mouse liver using AAV2/8 vectors. Mol Ther: J Am Soc Gene Ther 16:1081-1088

24. Guo P, El-Gohary Y, Prasadan K et al (2012) Rapid and simplified purification of recombinant adeno-associated virus. J Virol Methods 183:139-146

25. Zhang X, Xu A, Chung SK et al (2011) Selective inactivation of cJun NH2-terminal kinase in adipose tissue protects against dietinduced obesity and improves insulin sensitivity in both liver and skeletal muscle in mice. Diabetes 60:486-495

26. Lin Z, Tian H, Lam KS et al (2013) Adiponectin mediates the metabolic effects of FGF21 on glucose homeostasis and insulin sensitivity in mice. Cell Metab 17:779-789

27. Kim AY, Park YJ, Pan X et al (2015) Obesity-induced DNA hypermethylation of the adiponectin gene mediates insulin resistance. Nat Commun 6:7585

28. Nie T, Hui X, Gao X et al (2012) Adipose tissue deletion of Gpr116 impairs insulin sensitivity through modulation of adipose function. FEBS Lett 586:3618-3625
29. $\mathrm{Xu} \mathrm{A,} \mathrm{Wong} \mathrm{LC,} \mathrm{Wang} \mathrm{Y,} \mathrm{Xu} \mathrm{JY,} \mathrm{Cooper} \mathrm{GJ,} \mathrm{Lam} \mathrm{KS} \mathrm{(2004)}$ Chronic treatment with growth hormone stimulates adiponectin gene expression in 3T3-L1 adipocytes. FEBS Lett 572:129-134

30. Hibuse T, Maeda N, Funahashi T et al (2005) Aquaporin 7 deficiency is associated with development of obesity through activation of adipose glycerol kinase. Proc Natl Acad Sci U S A 102:1099310998

31. Thenen SW, Mayer J (1975) Adipose tissue glycerokinase activity in genetic and acquired obesity in rats and mice. Proc Soc Exp Biol Med 148:953-957

32. Huijsman E, van de Par C, Economou C et al (2009) Adipose triacylglycerol lipase deletion alters whole body energy metabolism and impairs exercise performance in mice. Am J Physiol Endocrinol Metab 297:E505-E513

33. Zhang X, Yeung DC, Karpisek M et al (2008) Serum FGF21 levels are increased in obesity and are independently associated with the metabolic syndrome in humans. Diabetes 57:1246-1253

34. Nakatsuji H, Kishida K, Sekimoto R et al (2014) Accumulation of adiponectin in inflamed adipose tissues of obese mice. Metab Clin Exp 63:542-553

35. Festuccia WT, Laplante M, Berthiaume M, Gelinas Y, Deshaies Y (2006) PPARgamma agonism increases rat adipose tissue lipolysis, expression of glyceride lipases, and the response of lipolysis to hormonal control. Diabetologia 49:2427-2436

36. Maeda N, Funahashi T, Shimomura I (2008) Metabolic impact of adipose and hepatic glycerol channels aquaporin 7 and aquaporin 9 . Nat Clin Pract Endocrinol Metab 4:627-634

37. Kosuga M, Henderson-MacLennan NK, Zhang YH, Huang BL, Dipple KM, McCabe ER (2011) Glycerol homeostasis and metabolism in glycerol kinase carrier mice. Mol Genet Metab 103:297299

38. Rodriguez A, Catalan V, Gomez-Ambrosi J, Fruhbeck G (2011) Aquaglyceroporins serve as metabolic gateways in adiposity and insulin resistance control. Cell Cycle 10:1548-1556

39. Lebeck J (2014) Metabolic impact of the glycerol channels AQP7 and AQP9 in adipose tissue and liver. J Mol Endocrinol 52:R165R178

40. Rodriguez A, Catalan V, Gomez-Ambrosi J et al (2011) Insulin- and leptin-mediated control of aquaglyceroporins in human adipocytes and hepatocytes is mediated via the $\mathrm{PI} 3 \mathrm{~K} / \mathrm{Akt} / \mathrm{mTOR}$ signaling cascade. J Clin Endocrinol Metab 96:E586-E597

41. Guo M, Chen F, Lin T et al (2014) Apelin-13 decreases lipid storage in hypertrophic adipocytes in vitro through the upregulation of AQP7 expression by the PI3K signaling pathway. Med Sci Monit: Int Med J Exp Clin Res 20:1345-1352

42. Kishida K, Shimomura I, Kondo H et al (2001) Genomic structure and insulin-mediated repression of the aquaporin adipose (AQPap), adipose-specific glycerol channel. J Biol Chem 276:36251-36260

43. Maeda N, Funahashi T, Hibuse T et al (2004) Adaptation to fasting by glycerol transport through aquaporin 7 in adipose tissue. Proc Natl Acad Sci U S A 101:17801-17806

44. Hara-Chikuma M, Sohara E, Rai T et al (2005) Progressive adipocyte hypertrophy in aquaporin-7-deficient mice: adipocyte glycerol permeability as a novel regulator of fat accumulation. J Biol Chem 280:15493-15496

45. Rodriguez A, Catalan V, Gomez-Ambrosi J, Fruhbeck G (2006) Role of aquaporin-7 in the pathophysiological control of fat accumulation in mice. FEBS Lett 580:4771-4776

46. Samuel VT, Shulman GI (2012) Mechanisms for insulin resistance: common threads and missing links. Cell 148:852-871

47. Tcatchoff L, Nespoulous C, Pernollet JC, Briand L (2006) A single lysyl residue defines the binding specificity of a human odorantbinding protein for aldehydes. FEBS Lett 580:2102-2108

48. Parlee SD, Lentz SI, Mori H, MacDougald OA (2014) Quantifying size and number of adipocytes in adipose tissue. Methods Enzymol 537:93-122 